\title{
Cross-sectional associations of meal size and meal timing with lipid biomarkers in a population-based cohort
}

Late night eating has been associated with a higher risk of cardiovascular mortality ${ }^{(1)}$ and breakfast skipping with unfavourable cardiovascular risk factors ${ }^{(2)}$. We have previously reported that a high energy breakfast is associated with lower cholesterol concentrations ${ }^{(3)}$. Here, we describe associations of meal size and meal timing with triglyceride (TG) and cholesterol concentrations, while considering time-of-day variation of these biomarkers.

The Norfolk-based European Prospective Investigation into Cancer and Nutrition (EPIC-Norfolk) recruited men and women, aged 39-79 y from GP practices between 1993-1998 ${ }^{(4)}$. Anthropometry was measured at health visits at which point non-fasting blood samples were taken (between 08:00-19:00) and serum cholesterol and TG concentrations determined. TGs were log-transformed. Those using lipid lowering medication were excluded as well as participants who reported illness or nightshifts. Participants completed a pre-structured 7-day diet diary consisting of eight recording sections: before breakfast (BB), breakfast (B), midmorning (MM), lunch (L), tea (T), dinner (D), evening (E) and 'unknown time' (U). We calculated mean reported energy intake (MJ/d) for each section, representing 'meal size'. Analysis of covariance was used to investigate associations of the energy content of individual meal sections with lipid concentrations (eight models per lipid), adjusted for: total energy intake (the beta of the meal section represents moving $1 \mathrm{MJ}$ of any section to the meal section of interest while keeping daily energy intake unchanged), hour of blood sampling, hours fasted, season, sex, age, physical activity, smoking, alcohol, education and BMI $(\mathrm{N}=22,068)$. Significant contributions to the model were determined by the F-test $(P<0 \cdot 05)$.

Mean (SD) energy intake at the eight recording sections were: $0.08(0 \cdot 15), 1 \cdot 25(0 \cdot 68), 0 \cdot 35(0.44), 2 \cdot 34(0 \cdot 90), 0 \cdot 37(0 \cdot 40), 2 \cdot 98(1 \cdot 06)$, $0.63(0.57), 0.23(0.34) \mathrm{MJ} / \mathrm{d}$ respectively; for cholesterol and TG these were $6 \cdot 19(1.17)$ and $1.81(1 \cdot 11)$ respectively. Moving $1 \mathrm{MJ}$ of energy to the meal section of interest was associated with higher lipid concentrations, apart from cholesterol at breakfast and TG at tea and dinner (top graphs). Only TG varied by blood sampling time for which significant interactions between sampling time and energy intake at breakfast and lunch were observed $(P<0.001$, bottom graphs).


The association between breakfast and cholesterol might be confounded by diet quality. Blood sampling time clarified associations between meal patterns and TG and may help in understanding diet-disease associations, in which circadian variation could be included.

1. Cahill LE, Chiuve SE, Mekary RA et al. (2013) Circulation 128, 337-43.

2. St-Onge M-P, Ard J, Baskin ML et al. (2017) Circulation 135, e96-e121.

3. Lentjes M, Mulligan AA, Welch AA et al. (2013) Proc Nutr Soc, 72, E297.

4. Day N, Oakes S, Luben R et al. (1999) Br. J. Cancer 80, Suppl. 1, 95-103. 